\title{
Verification, Validation, and Testing of Kinetic Mechanisms of Hydrogen Combustion in Fluid-Dynamic Computations
}

\author{
Victor P. Zhukov \\ Institute of Space Propulsion, German Aerospace Center (DLR), Lampoldshausen, 74239 Hardthausen, Germany \\ Correspondence should be addressed to Victor P. Zhukov, victor.zhukov@dlr.de \\ Received 21 May 2012; Accepted 13 June 2012 \\ Academic Editors: F. Liu and B. Yu \\ Copyright ( $) 2012$ Victor P. Zhukov. This is an open access article distributed under the Creative Commons Attribution License, \\ which permits unrestricted use, distribution, and reproduction in any medium, provided the original work is properly cited. \\ A one-step, a two-step, an abridged, a skeletal, and four detailed kinetic schemes of hydrogen oxidation have been tested. A new \\ skeletal kinetic scheme of hydrogen oxidation has been developed. The CFD calculations were carried out using ANSYS CFX \\ software. Ignition delay times and speeds of flames were derived from the computational results. The computational data obtained \\ using ANSYS CFX and CHEMKIN, and experimental data were compared. The precision, reliability, and range of validity of the \\ kinetic schemes in CFD simulations were estimated. The impact of kinetic scheme on the results of computations was discussed. \\ The relationship between grid spacing, time step, accuracy, and computational cost was analyzed.
}

\section{Introduction}

In the last decade various commercial software packages such as ANSYS CFX [1], Fluent [2], and Star-CD [3], etc are widely used as a tool for solving computational fluid-dynamics (CFD) problems. According to the website http://www.top500.org, computational capabilities are increasing by a factor of 100 every eight years. The explosive growth of computational power has allowed CFD simulations using more and more complicated physical models to be completed on small clusters of computers or even desktop computers (without using high-power expensive computers). Modern CFD simulations can be multicomponent, multiphase, and multidomain. Heat, mass, and radiation transfer as well as chemical processes can be taken into account in calculations. The increased amount of model assumptions and parameters is an significant source of errors and faults.

The processes of verification and validation are very important in CFD [4]. They are ground steps in obtaining a numerical solution (Figure 1). The validation should be completed before obtaining the desired numerical results while the verification should be completed before the validation. Normally, the whole numerical model, which includes equations of fluid dynamics, equation of state, and the model of turbulence, is already verified by the developer of the CFD code and the user should verify only its own user defined models.

Our ultimate aim is the modeling of the flow in a rocket combustion chamber. For this purpose, we cannot rely on the predefined numerical model, but should use the models which take into account the specifics of this complicated problem. Here, we are focusing on the usage of the chemical kinetic models of hydrogen combustion. In most cases, the assumption of thin flame (infinitely fast chemical reactions) gives reliable results, so there is no actual need to use the detailed kinetic mechanisms in CFD simulations. However, the assumption of thin flame is not completely satisfied in rocket combustion chamber where the turbulence is very high. Therefore the model of the chemical kinetics should be used for modeling combustion in rocket engine, after being verified and validated.

In our case, the verification can be done through the comparison with Chemkin [5] which solves a system of kinetic equations. This gives us a chance to find and eliminate misprints and to prove that numerical parameters, for example, time step and grid do not determine the solution. The next step should be the validation. After entering into a CFD code a chemical kinetic model became a part of large physical-chemical numerical model. Generally, kinetic mechanisms are already validated extensively by their 


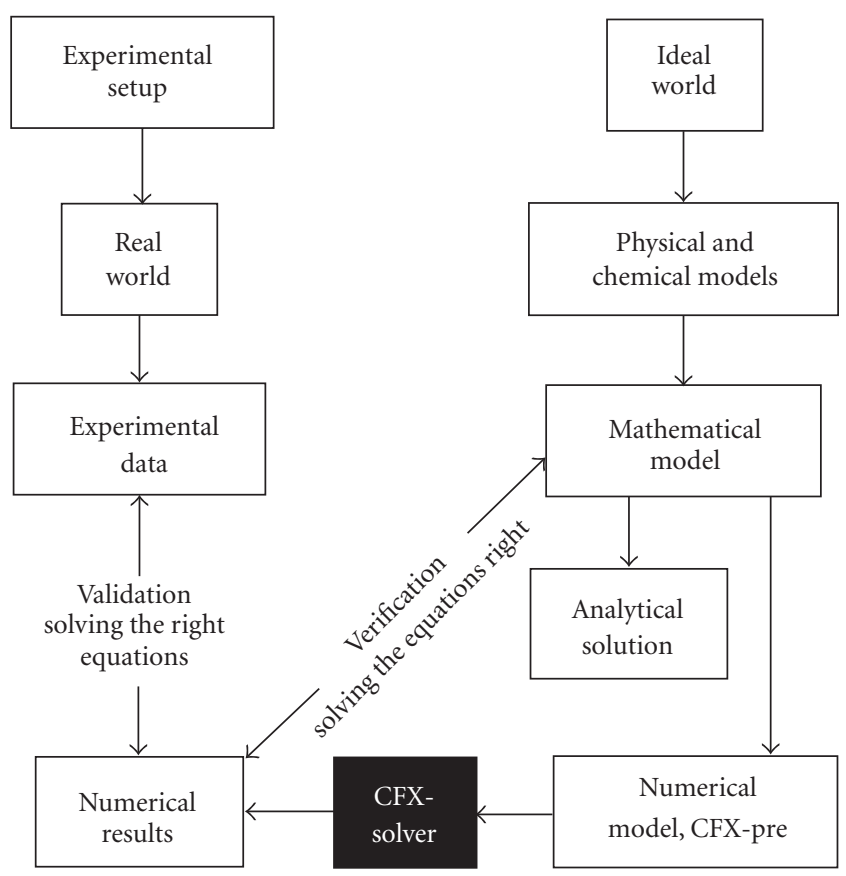

FIGURE 1: Logic scheme of validation and verification.

authors, but after the implementation of the chemical kinetic model the CFD numerical model needs the validation. Of course chemical reactions drive combustion, but indeed combustion processes depend on heat and mass transfer too. Although turbulence model, equations of state, transport coefficients, and chemical kinetic mechanism can be validated separately, the resulting physical-chemical model needs the final validation as a whole.

Probably the first example of the verification and the validation of hydrogen reaction mechanism in CFD simulations is the work by Mani et al. [6]. A supersonic flow in a constant-area channel was simulated. The employed kinetic scheme consisted of 8 reactions without the kinetics of the peroxides. The supersonic combustion of a hydrogenair mixture at a high temperature was modeled. The simple kinetic scheme reproduced the experimental data properly which is not surprising when the initial temperature is about $1400 \mathrm{~K}$.

In 1988 Jachimowski [7] modeled scramjet combustion using of a hydrogen-air reaction mechanism. The simulations of hydrogen combustion were computed with parameters related to flight speeds of Mach 8, 16, and 25. The hydrogen-air reaction mechanism consisted of 33 reactions, while the detailed hydrogen-oxygen kinetics consisted of 20 reactions. In his study, it was shown that chemical kinetics of $\mathrm{HO}_{2}$ affects the results at all of the studied Mach numbers. Later Eklund and Stouffer [8] carried out the 3D CFD simulation of a flow in a supersonic combustor. They tested two kinetic models: the detailed model by Jachimowski and the model abridged from the detailed one. This model was obtained by cutting the kinetics of $\mathrm{HO}_{2}$ and $\mathrm{H}_{2} \mathrm{O}_{2}$. The new model consists of only 7 reactions between 6 species plus bath gas $\mathrm{N}_{2}$. This abridged model is very extensively cited and used due to its low computational power requirements relative to detailed kinetic models.

Kumaran and Babu [9] studied the effect of chemical kinetic models on CFD calculations. They modeled a compressible, turbulent, and reacting flow, which simulates the supersonic combustion of hydrogen in the jet engine of hypersonic projectile. The idea of their work is to compare the results obtained using the detailed kinetic model with the previous results obtained with a single-step kinetics. In their previous study, the difference between the numerical results and the experimental data was attributed to the inadequacy of the one-equation turbulence model and the one-step chemistry. The simulations with the use of the detailed kinetic mechanism predicted higher combustion efficiency than the calculations using the one-step model. The comparison of wall static pressure from the experiments and the numerical simulations showed that the detailed and the single-step chemistry produce similar results and accurately predict the positions of the pressure peaks, but they fail to predict the values of pressure peaks. All the numerical simulations were carried out using the software package Fluent [2].

The use of a detailed mechanism should give a more precise estimation of the main thermodynamic parameters: temperature and pressure, and provide the distribution of the intermediates: $\mathrm{OH}, \mathrm{H}_{2} \mathrm{O}_{2}$, and so forth. Generally detailed kinetic models provides much more information about combustion processes, but requires an additional CPU power.

In the previously discussed work, Kumaran and Babu used the kinetic mechanism of Stahl and Warnatz [17] published in 1991 as the reference detailed mechanism. This mechanism became a little bit old after publication of works $[18,19]$ published in 2002. In these works, the refined data on the rate constant of reaction (R9) [18]:

$$
\mathrm{H}+\mathrm{O}_{2}+\mathrm{M} \longrightarrow \mathrm{HO}_{2}+\mathrm{M}
$$

and the enthalpy of formation of $\mathrm{OH}$ [19] were reported. Since radicals $\mathrm{OH}$ and $\mathrm{HO}_{2}$ play the essential role in hydrogen oxidation, all kinetic mechanisms developed before 2002 should be revised. In the current work the several detailed hydrogen kinetic mechanisms are tested. One is "old" [14], while three others $[13,16,20]$ were released after 2002. Besides the outdated thermodynamic data and rate constants the "old" mechanism by Gutheil et al. [14] has reaction:

$$
\mathrm{H}_{2}+\mathrm{O}_{2} \longrightarrow \mathrm{OH}+\mathrm{OH}
$$

The usage of this reaction has became marginal nowadays, for example: it is not included in the modern mechanisms $[13,15,20]$ considered below (by this reason, this reaction does not have a reference number, but is labeled "(RX)"). The "old" mechanism has been tested in order to see the difference from the updated mechanisms.

Konnov [20] reported recently about "remaining uncertainties in the kinetic mechanism of hydrogen combustion." He studied the detailed hydrogen combustion mechanism. Konnov found two groups of the uncertainties. The first 
group is associated with the set of chemical reactions in the hydrogen-oxygen system. Not all of the possible reactions are included in the kinetic mechanisms, and the set of reactions varies from one mechanism to another. Thereby, there is no one conventional set of reactions, which describes combustion of hydrogen comprehensively, and this problem is still open. The second group of uncertainties relate to the uncertainties in the rate constants of the employed reactions. Some of them are not well defined or the experimental data on them are controversial. It should be noted that the hydrogen kinetic mechanism developed by Konnov [15] has the slightly different set of reactions to other extensively cited mechanisms such as Princeton University [21], National University of Ireland, Galway [13], and so forth.

Shatalov et al. [22] carried out the analysis of several detailed kinetic mechanisms of hydrogen combustion: the mechanism by Konnov [20] and mechanisms by other authors. Shatalov et al. [22] noted that the use of reaction RX does not have a sense, because another parallel channel:

$$
\mathrm{H}_{2}+\mathrm{O}_{2} \longrightarrow \mathrm{H}+\mathrm{HO}_{2}
$$

has the significantly greater rate constant (in 50 and more times). On the other hand, the use of reaction (RX) helps a lot to fit experimental data. They pointed out also that below $1100 \mathrm{~K}$ reaction (R9) affects the ignition delay time significantly.

There are two very recent hydrogen reaction mechanisms from Princeton and Stanford universities [16, 23]. Both are validated against the latest experimental data. For our applications (rocket combustion), the experimental data of Burke et al. [24] (the same team as [16]) is the most interesting. This experimental data represents the measurements of the burning velocities in $\mathrm{H}_{2} / \mathrm{O}_{2} / \mathrm{He}$ mixtures at pressures of 1$25 \mathrm{~atm}$. These measurements are the only available data for hydrogen at high pressure. The comparison of these recent mechanisms with the experimental data showed that the mechanism by Burke et al. [16] has a better agreement with experimental data at high pressure than mechanism [23]. Therefore, only the mechanism by Burke et al. [16] has been chosen for the tests in this work.

A study similar to the current work was carried out by Gerlinger et al. [25]. The colleagues studied several hydrogen/air reaction mechanisms including multistep schemes $[7,8,13]$ and one-step mechanism by Marinov et al. [11]. The study is focused in the application of reaction mechanisms in the simulation of supersonic combustion. The mechanisms were validated against ignition delay times. In the validation all mechanism showed similar results except for the one-step mechanism [11], which do not capture the non-Arrenius behavior of the experimental data. The authors simulated supersonic combustion with different mechanisms and compared the results with experimental data. The influence of time step and numerical grid size was also examined. The numerical results showed that the results were dependent on the sensitivity to the time step and the grid density. Finally the authors conclude that the one-step mechanism [11] is not appropriate, while the mechanism by O'Conaire et al. [13] is more precise than other multistep mechanisms.
While examples of successful verification, validation, and application of hydrogen reaction mechanisms in CFD simulation of supersonic combustion ramjets exist, the problem of the CFD simulation of hydrogen combustion in rocket engines is not closed. Scramjet is a specific case and the results obtained for supersonic combustion cannot be extended to the case of rocket engines. Combustion in rocket engines has its own characteristic features such as high pressures (50-250 atm), the wide span of temperatures from $100 \mathrm{~K}$ to $3500 \mathrm{~K}$, and the absence of dilutant (nitrogen). For scramjets, the verification and validation can be done by directly simulating a supersonic combustion directly, while it is not yet possible to do the same by simulating combustion in rocket engines. In recent work, Tucker et al. [26], the group of researchers from five research centers, made the CFD simulations of a flow in a combustion chamber. Each participant simulated the same test case using their own methodology. The test case was a subscale rocket engine with 1.5 inch inner diameter, and one coaxial injector. The combustion chamber had an axial symmetry, which allowed to carry out the comparison of $2 \mathrm{D}$ and $3 \mathrm{D}$ modeling. Tucker et al. compared steady Reynolds-Average NavierStockes (RANS), Unsteady Reynolds-Average Navier-Stockes (URANS), and three different large eddy simulation (LES) models with the experiment. The comparison showed that all approaches give noticeably different results and that only one approach (LES - stochastic reconstruction model) produced results which were comparable to the experimental data. The most precise modeling results were obtained with the finest mesh of $255 \cdot 10^{6}$ cells and using the computational power of 2 million cumulative CPU hours. However, it is not totally clear how the initial model assumptions affected the accuracy of the final results, and what assumption or parameter impaired the other models. Such a comparison is very important from the practical point of view as the computational cost and precision vary strongly from one numerical model to another.

The performance of the chemical kinetic models of hydrogen oxidation in CFD simulations has been estimated in the current work. The aims of the work are the ranking of the selected hydrogen kinetic models and the development of the verification, validation, and ranking procedures. The performance of the kinetic models is assessed against the experimental data on hydrogen ignition [10] and hydrogen flame speed [27]. The CFD simulations are carried out using complex physical models. There is currently no conventional methods for verifying combustion models as part of the whole physical-chemical model, and no conventional methods for verifying and validating the physical model itself. In such conditions, the all-round verification is simply obligatory for the CFD modeling. The simulations have a secondary aim to estimate the validity region within the space of the computational parameters: mesh, computational scheme, and time step. The precision (the difference between calculations and experiments) and the computational cost (required CPU time) were estimated for each test case. A global reaction model [11], a two-step scheme [12], an abridged Jachimowski's model [8], a new skeletal mechanism, and four detailed hydrogen mechanisms $[13,14,16$, 
20] have been tested. The results indicate which chemical kinetic scheme and parameters should be used along with the required compting power for the computations. The work is the first step before conducting CFD simulations of the experiments carried out at our test facility [28, 29].

\section{Skeletal Kinetic Model}

In our case as well as in other, CFD simulations the numerical and the physical models have a lot of parameters and need the debugging before getting the final solution. Parameters, which are not connected with the problem directly, for example, time step, can seriously obstruct obtaining a solution. Detailed kinetic mechanisms make CFD models too heavy for the debugging. On the other side, global reaction models do not include the minor species and do not allow to do the all-round debugging (validation and verification of the properties of $\mathrm{HO}_{2}, \mathrm{H}_{2} \mathrm{O}_{2}$, etc.).

In this work, a skeletal kinetic scheme has been developed, which has the same set of species as detailed hydrogen mechanisms, but the reduced set of reactions. This light scheme sped up the formulation of the computational problem. The problem definition requires to perform the certain set of the calculations. Different meshes and the models of diffusion and thermal conductivity were tried before getting the final results. The light skeletal scheme reduced the amount of the expended CPU hours at the preliminary stage. The new scheme fills the gap between abridged Jachimowski's model [8], which has 7 reactions and 6 species, and the detailed hydrogen mechanisms [1315] (19-21 reactions, 8 species, and bath gases) as well as it allows to separate the influence of the amount of reactions and species for the calculating speed and the computational accuracy.

The new scheme was developed from the skeletal model by Kreutz and Law [30]. Their skeletal kinetic model has 9 unidirectional reactions and 8 species. Considering $\mathrm{H}_{2} / \mathrm{O}_{2}$ system, it may assume that the set of 9 species: $\mathrm{H}_{2}, \mathrm{O}_{2}$, $\mathrm{H}_{2} \mathrm{O}, \mathrm{H}, \mathrm{O}, \mathrm{OH}, \mathrm{HO}_{2}, \mathrm{H}_{2} \mathrm{O}_{2}$, and bath gas is complete and other species $\left(\mathrm{O}_{3}, \mathrm{OH}^{-}, \mathrm{OH}^{*}(\mathrm{~A})\right.$, etc. $)$ can play role only in marginal cases. It is necessary to note that at high pressures, which is the case of rocket combustion chambers, the chain branching proceeds via the formation of $\mathrm{HO}_{2}, \mathrm{H}_{2} \mathrm{O}_{2}$ radicals due to the high rates of the recombination processes [31]. For example, reaction:

$$
\mathrm{H}+\mathrm{O}_{2} \longrightarrow \mathrm{OH}+\mathrm{O}
$$

which is the most important in atmospheric hydrogen flames, is suppressed by reaction (R9) at pressures above 50 bar. The model by Kreutz and Law [30] has a 5 times smaller set of reactions as detailed hydrogen mechanisms and can adequately predict the ignition delay times and the ignition limits. On the other hand, the scheme consists of the irreversible reactions, which means that the concentrations of species never reach the equilibrium state. The afterburning processes are omitted, which is not important during ignition, but leads the mispredictions of species profiles. By this reason, the reaction set was extended by 6 reactions from detailed hydrogen model [13]. The reaction of the quadratic recombination of $\mathrm{HO}_{2}$ radicals:

$$
\mathrm{HO}_{2}+\mathrm{HO}_{2} \longrightarrow \mathrm{H}_{2} \mathrm{O}_{2}+\mathrm{O}_{2}
$$

was substituted by reactions $\mathrm{R} 11$ and R13, see Tables 1 and 2. Such extension increases the computational weight of the model, but it increases the adequacy of the model as well. The new added reactions involve the processes of radical recombinations, which are important in a postflame zone.

\section{Calculations}

The CFD calculations have been done with the use of the ANSYS CFX 11 solver [1], which utilizes the finite volume element method (FVEM). The meshes have been created using ANSYS ICEM software. The choice of the software is given by an adherence to the compatibility of the computer data and the design documentation.

Two types of tests (simulations) have been done in the work. The first test case is a quasi-0-dimensional simulation of hydrogen ignition to verify and validate the models against the experimental data on ignition delay times [10]. The second test case is an 1-dimensional simulation of hydrogen flame propagation to test the models against the data on the speeds of laminar flame [27].

An ignition in a perfect adiabatic constant volume reactor has been modeled as the quasi-0D problem. By the formulation, the problem is dimensionless, but by the settings of calculations, it is 3D. The computational domain represents eighth part of the $1 \mathrm{~mm}$ sphere with rigid adiabatic walls. The mesh consists of 21 nodes and 38 tetrahedron elements. At the initial moment, the whole domain is filled with a stoichiometric hydrogen-air $\left(0.79 \mathrm{~N}_{2}+0.21 \mathrm{O}_{2}\right)$ mixture at pressure of $1 \mathrm{~atm}$ and temperature in the range of 900-1400 K. The problem has been solved as a transient task, that is, the time evolution of gas conditions has been sought. The laminar model (unsteady Navier-Stokes equations) was employed, but also one series of simulations were carried out using the $k-\epsilon$ turbulence model. The comparison of results shows that both models (the laminar and the $k-\epsilon$ model) give the same results in this task. The task was imposed in such way that the results should be independent of the choice of turbulence model. Indeed, the stagnant homogeneous gas mixture is surrounded with the adiabatic rigid walls, so the gas inside the sphere should be stagnant all time. The object of these calculations is the estimation of the ignition delay times and the comparison of the calculated delay times with the data from shock tube experiments [10], see Figure 2. In the calculations, the ignition delay times were defined as the time of a temperature increase up to $500 \mathrm{~K}$ relative to the initial temperature.

During the $1 \mathrm{D}$ tests, a freely propagating hydrogen flame has been modeled. The computational domain consists of 1604 nodes and 400 rectangular prism elements. All elements are placed along one axis so that the thickness of the domain equals to one element in two other coordinate axes. The 
TABLE 1: New skeletal mechanism.

\begin{tabular}{|c|c|c|c|c|c|c|}
\hline No & $\begin{array}{l}\text { Reference } \\
\text { no }\end{array}$ & Reaction & $A$ & $n$ & $E_{a}$ & Reference \\
\hline 1 & $\mathrm{R} 1$ & $\mathrm{H}+\mathrm{O}_{2} \rightarrow \mathrm{OH}+\mathrm{O}$ & $1.91 e+14$ & 0.0 & 16.44 & {$[30]$} \\
\hline 2 & $\mathrm{R} 2$ & $\mathrm{H}_{2}+\mathrm{O} \rightarrow \mathrm{H}+\mathrm{OH}_{2}$ & $5.08 e+4$ & 2.67 & 6.292 & {$[30]$} \\
\hline 3 & R3 & $\mathrm{H}_{2}+\mathrm{OH} \rightarrow \mathrm{H}+\mathrm{H}_{2} \mathrm{O}$ & $2.16 e+8$ & 1.51 & 3.43 & {$[30]$} \\
\hline 4 & R5 & $\mathrm{H}_{2}+\mathrm{M} \leftrightarrow \mathrm{H}+\mathrm{H}+\mathrm{M}$ & $4.57 e+19$ & -1.4 & 105.1 & {$[13]$} \\
\hline 5 & R6 & $\mathrm{O}+\mathrm{O}+\mathrm{M} \leftrightarrow \mathrm{O}_{2}+\mathrm{M}$ & $6.17 e+15$ & -0.5 & 0.0 & {$[13]$} \\
\hline 6 & R7 & $\mathrm{H}+\mathrm{O}+\mathrm{M} \rightarrow \mathrm{OH}+\mathrm{M}$ & $4.72 e+18$ & -1.0 & 0.0 & {$[13]$} \\
\hline 7 & $\mathrm{R} 8$ & $\mathrm{H}+\mathrm{OH}+\mathrm{M} \rightarrow \mathrm{H}_{2} \mathrm{O}+\mathrm{M}$ & $4.5 e+22$ & -2.0 & 0.0 & {$[13]$} \\
\hline 8 & R9 & $\mathrm{H}+\mathrm{O}_{2}+\mathrm{M} \rightarrow \mathrm{HO}_{2}+\mathrm{M}$ & $6.17 e+19$ & -1.42 & 0.0 & {$[30]$} \\
\hline 9 & $\mathrm{R} 10$ & $\mathrm{H}+\mathrm{HO}_{2} \rightarrow \mathrm{H}_{2}+\mathrm{O}_{2}$ & $1.66 e+13$ & 0.0 & 0.82 & {$[13]$} \\
\hline 10 & $\mathrm{R}-10$ & $\mathrm{H}_{2}+\mathrm{O}_{2} \rightarrow \mathrm{H}+\mathrm{HO}_{2}$ & $3.68 e+13$ & 0.203 & 54.46 & {$[30]$} \\
\hline 11 & $\mathrm{R} 11$ & $\mathrm{H}+\mathrm{HO}_{2} \rightarrow \mathrm{OH}+\mathrm{OH}$ & $1.69 e+14$ & 0.0 & 0.87 & {$[30]$} \\
\hline 12 & $\mathrm{R} 13$ & $\mathrm{OH}+\mathrm{HO}_{2} \rightarrow \mathrm{H}_{2} \mathrm{O}+\mathrm{O}_{2}$ & $2.89 e+13$ & 0.0 & -0.5 & {$[13]$} \\
\hline 13 & $\mathrm{R} 15$ & $\mathrm{H}_{2} \mathrm{O}_{2}+\mathrm{M} \rightarrow \mathrm{OH}+\mathrm{OH}+\mathrm{M}$ & $1.2 e+17$ & 0.0 & 45.5 & {$[30]$} \\
\hline 14 & $\mathrm{R}-17$ & $\mathrm{H}_{2}+\mathrm{HO}_{2} \rightarrow \mathrm{H}+\mathrm{H}_{2} \mathrm{O}_{2}$ & $3.42 e+12$ & 0.202 & 27.12 & {$[30]$} \\
\hline
\end{tabular}

$k=A \cdot T^{n} \cdot \exp \left(-E_{a} / R T\right)$; units: mol, $\mathrm{cm}^{3}, \mathrm{~K}$, and kcal; thermodynamic data [5]; the reverse rate constants (R5, R6) are calculated from the forward rate constants through the equilibrium constants.

TABLE 2: Efficiency factors for third body term.

\begin{tabular}{lcccccccc}
\hline Reference no & $\mathrm{H}$ & $\mathrm{H}_{2}$ & $\mathrm{H}_{2} \mathrm{O}$ & $\mathrm{H}_{2} \mathrm{O}_{2}$ & $\mathrm{HO}_{2}$ & $\mathrm{O}$ & $\mathrm{O}_{2}$ & $\mathrm{OH}$ \\
\hline R5 & 1.0 & 2.5 & 12 & 1.0 & 1.0 & 1.0 & 1.0 & 1.0 \\
R6 & 0.83 & 2.5 & 12 & 1.0 & 1.0 & 0.83 & 1.0 & 1.0 \\
R7 & 0.75 & 2.5 & 12 & 1.0 & 1.0 & 0.75 & 1.0 & 1.0 \\
R8 & 1.0 & 0.73 & 12 & 1.0 & 1.0 & 1.0 & 1.0 & 1.0 \\
R9 & 1.0 & 2.5 & 12 & 1.0 & 1.0 & 1.0 & 1.0 & 1.0 \\
R15 & 1.0 & 2.5 & 12 & 1.0 & 1.0 & 1.0 & 1.0 & 1.0 \\
\hline
\end{tabular}

mesh spacing equals to $5 \mu \mathrm{m}$ in the direction of the flame propagation. The separate study of the influence of the grid spacing was carried out where the spacing was varied from $0.2 \mu \mathrm{m}$ to $200 \mu \mathrm{m}$. The domain represents the rectangular with symmetry boundary conditions on the side walls. The domain has one inlet and one outlet (on the side opposite to inlet). At the outlet, static pressure is specified and equals $1 \mathrm{~atm}$. At the inlet, a hydrogen-air $\left(0.79 \mathrm{~N}_{2}+0.21 \mathrm{O}_{2}\right)$ mixture at $298 \mathrm{~K}$ and $1 \mathrm{~atm}$ flows inside the domain. The velocity of the mixture is specified at the inlet in the range of 0.5$3.5 \mathrm{~m} / \mathrm{s}$ so that the velocity of the flame front reaches a small value in the laboratory system of coordinates. The mixture composition was varied from equivalence ratio of $\mathrm{ER}=0.5$ to $\mathrm{ER}=4.5$. The simulations were run as a transient task. A stationary burning velocity was sought. The laminar model was employed, also one series of tests were carried out using the $k-\epsilon$ turbulence model. Speed of flame depends essentially on the transport properties of gas, so the temperature dependent thermal conductivity and diffusion coefficients were used. Thermal diffusion was not taken into account. The system of the governing equations in ANSYS CFX does not assume mass fluxes caused by temperature gradients.

\section{Results and Discussion}

4.1. Verification: Comparison with CHEMKIN. The both tasks were also solved in CHEMKIN II [5]. The results of the simulations with the help of CHEMKIN II were used as a reference data. CHEMKIN is very widely used for solving chemical kinetic problems, where the computational problem is formulated as solving of a system of ordinary differential equations. Indeed, ANSYS CFX allows to specify the properties of a system by the different ways, while in CHEMKIN task is set in the one prescribed format. CHEMKIN uses the modified Arrenius form for rate coefficients:

$$
k=A \cdot T^{n} \cdot \exp \left(-\frac{E_{a}}{\mathrm{RT}}\right) .
$$

The thermodynamic functions: enthalpy, entropy, and heat capacity are calculated using the NASA polynomial forms in CHEMKIN. During the calculations in ANSYS CFX, the same equations were employed for the rate constants, the thermodynamic functions, and the equations of states, so the comparisons of the results obtained using the different software have a sense. The results of the comparisons are 


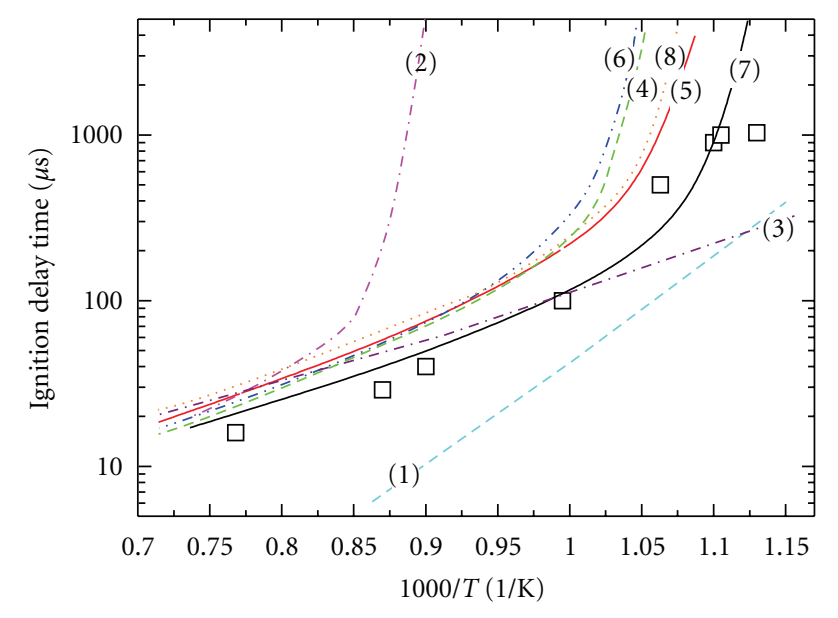

FIgURE 2: Ignition delay times of a stoichiometric hydrogen-air mixture at $1 \mathrm{~atm}$. Squares-experimental data [10]; (1) dash cyan line-Marinov et al. [11]; (2) dash dot magenta line-Lee and Kim [12]; (3) dash dot purple line-the abridged Jachimowski's model [8]; (4) dash line green-Zhukov (this work); (5) solid red lineO'Conaire et al. [13]; (6) dash dot dot line blue-Gutheil et al. [14]; (7) solid black line (the closest to exp. data)—Konnov [15]; (8) dot orange line-Burke et al. [16].

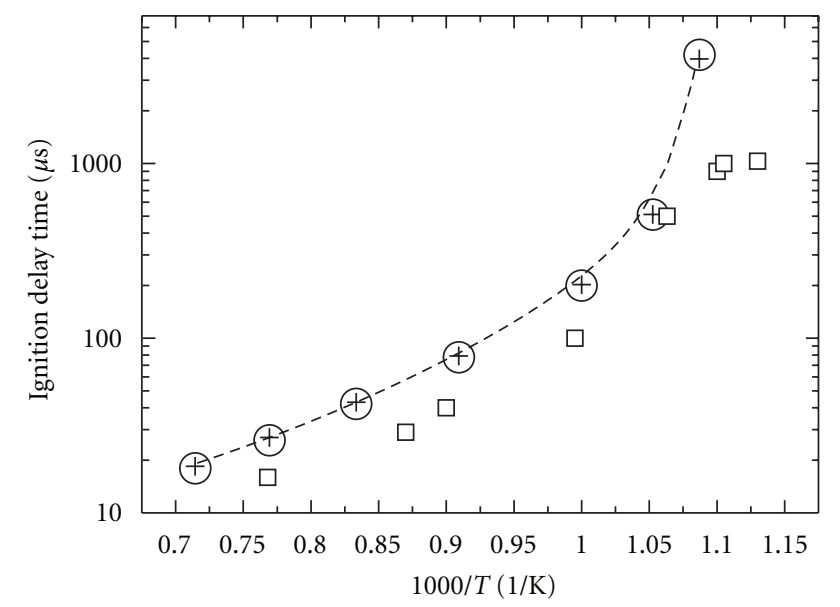

FIgURE 3: Comparison of the simulating data obtained using ANSYS CFX and CHEMKIN. Squares - the experimental ignition delay times of a stoichiometric hydrogen-air mixture at 1 atm [10]; the kinetic model by O'Conaire et al. [13]: dash line (B-spline) and crosses-ANSYS CFX, big circles-CHEMKIN.

depicted in Figures 3 and 4, where ANSYS CFX shows the agreement with CHEMKIN.

Using CHEMKIN, the ignition delay times were calculated in the assumptions of constant volume and adiabatic walls. In this case, the problem definitions (the sets of boundary conditions and kinetic equations) correspond to each other in ANSYS CFX and CHEMKIN. As a consequence, the results of the simulations using ANSYS CFX agree fully with the computations in CHEMKIN, see Figure 3. Indeed, it is necessary to note that CFX solves the 3-dimensional NavierStokes equations while CHEMKIN uses the 0-dimensional equation of energy conservation.

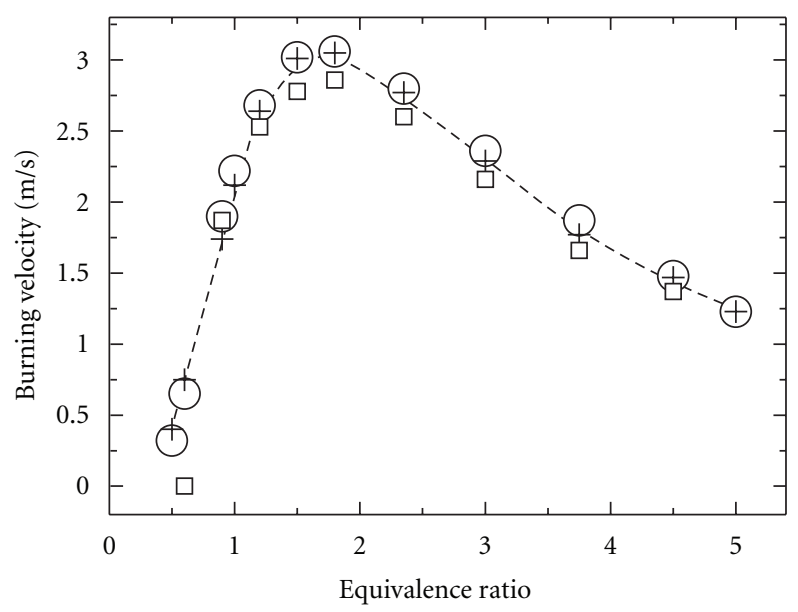

FIGURE 4: Comparison of the simulating data obtained using ANSYS CFX and CHEMKIN. Squares - the experimental burning velocities of a hydrogen-air mixture at $1 \mathrm{~atm}$ and $298 \mathrm{~K}$ [27]; the kinetic model by Konnov [15]: dash line (B-spline) and crossesANSYS CFX, big circles-CHEMKIN.

The next step is the modeling of freely propagating laminar flame. Zeldovich-Frank-Kamenetskii equation, which connects flame velocity and reactivity, gives us a clear view on the problem:

$$
u_{\mathrm{lam}}=\sqrt{\frac{\alpha}{\tau}}
$$

where $\tau$ is the chemical time scale in reaction zone, and $\alpha$ is the coefficient of temperature conductivity, which summarizes the effect of diffusion and heat conductivity through Lewis number Le $=1$. In contrast to the previous case, kinetic and transport properties have an equal importance in flame propagation.

The flame speeds were estimated using ANSYS CFX and PREMIX [32], Figure 4. PREMIX is a subroutine of the CHEMKIN which computes species and temperature profiles in steady-state laminar flames. The transport properties were estimated using TRANFIT: the another part of the CHEMKIN collection. Thermal conductivity, viscosity, and diffusion coefficients are estimated from the parameters of the Lennard-Jones potential and the dipole moment of species. The same temperature depended coefficients of thermal conductivity, viscosity, and binary diffusion were used in PREMIX and ANSYS CFX, but diffusion fluxes in multicomponent mixture were approximated by a different way.

The specific of $\mathrm{H}_{2}-\mathrm{O}_{2}$ system is that the properties of hydrogen (the lightest gas) are distinguished strongly from the properties of other species within the system. By default, ANSYS CFX estimates the transport properties in the mixture of gases by an inappropriate way for $\mathrm{H}_{2}-\mathrm{O}_{2}$ system, where the influence of the fuel on the transport properties is important. It calculates the coefficient of thermal conductivity and viscosity of gas mixture using a mass averaging, and the coefficients of diffusion are calculated from the mixture bulk viscosity. The problem becomes significant in the case 
of combustion in rocket engine where the mixture is not diluted by nitrogen. This problem can be resolved in ANSYS CFX using CFX Expression Language (CEL) and setting all coefficients by user as it was done in this work.

In $\mathrm{H}_{2}-\mathrm{O}_{2}$ system, the diffusion coefficients vary from one component to other in $\sim 6$ times: $D_{\mathrm{O}_{2}} / D_{\mathrm{H}} \approx \sqrt{\left(\mu_{\mathrm{O}_{2}} / \mu_{\mathrm{H}}\right)} \approx$ $\sqrt{32}$. Even in the simplest case, where only $\mathrm{H}_{2}, \mathrm{O}_{2}$, and $\mathrm{H}_{2} \mathrm{O}$ are taken into account, the gas mixture can not be assumed as a binary mixture or as a solution of light gas in heavy gas due to the high fractions of $\mathrm{H}_{2}$ and $\mathrm{H}_{2} \mathrm{O}$ and the differences in the diffusion coefficients. In the current work, the diffusion coefficients are calculated by the empirical formula:

$$
D_{i}=\frac{1-w_{j}}{\sum X_{j} / D_{i j}},
$$

where $w_{i}$ is the mass fraction of $i$-species; $X_{j}$ is the mole fraction of $j$-species; $D_{i j}$ is the binary diffusion coefficient [33]. After that, the diffusion coefficients of individual species are put into the equation which is responsible for the transport in CFX:

$$
\rho_{i}\left(U_{\text {mix }}-U_{i}\right)=-\frac{D_{i}}{\rho_{\text {mix }}} \frac{\partial \rho}{\partial x},
$$

where $\rho_{i}\left(U_{\text {mix }}-U_{i}\right)$ is the relative mass flux of $i$-species. The equation is not solved for one constraint component (in our case nitrogen), whose mass fraction is calculated from the constraint that the sum of mass fractions of all species equals 1. PREMIX (CHEMKIN) uses a more accurate definitions of the diffusion and the thermal conductivity in gas mixture and takes into account the thermal diffusion of $\mathrm{H}$ and $\mathrm{H}_{2}$. There are two options: "mixture-averaged properties" and "multicomponent properties" in PREMIX. "Mixture-averaged" option, which was used here, employ (4), but does not have a constraint species and employs an additional term-correction velocity, which makes the net species diffusion flux equal to zero. "Multicomponent" formulation uses the method described by Dixon-Lewis [34], where the coefficients are computed from the solution of a system of equations defined by the $L$ matrix.

The flame velocities obtained with the use of CFX and CHEMKIN coincide practically with each other. The difference in the results, which is small (Figure 4), should be associated with the distinction between the formulations of the diffusion fluxes. Coffee and Heimerl [35] compared various methods of approximating the transport properties of premixed laminar flames, in particular the methods which have been used in CFX and CHEMKIN. They found that the difference in flame speed is small for these methods, but the method, which is employed in CHEMKIN, is more accurate than the method with constrained species (CFX), which is inaccurate in computing the diffusion velocity for constrained species. As for the comparison with experimental data, it was shown in recent work [36] that such small overshooting around the stoichiometry, which is observed in Figure 4, results from the neglecting Soret effect (thermodiffusion).

In our case (laminar hydrogen-air flame at $1 \mathrm{~atm}$ ), the propagation of the flame is supported essentially by the diffusion $\mathrm{H}_{2}$ into flame zone and the diffusion of $\mathrm{H}$ into preflame zone. That is why it is important to estimate accurately the diffusion term. In Figure 5, we can see the effect of the transport properties on laminar flame. The maximum of the flame speed is shifted to the higher equivalent ratios where the diffusive fluxes of $\mathrm{H}$ and $\mathrm{H}_{2}$ are higher.

4.2. Validation and Testing. Let us consider the results of the first " $0 \mathrm{D}$ " test case, which is depicted in Figure 2. The detailed models [13-16] agree with experimental data well, while the nondetailed kinetic models [11, 12], abridged Jachimowski's model [8], and the new skeletal model have the agreement with experimental data only in the limited range. The kinetic model by Konnov [20] agrees with experimental data better than other models. In Figure 2, it is possible to see the transition from high-temperature kinetics to low-temperature kinetics around $950 \mathrm{~K}$. Generally, the models show the common trend: more details-higher accuracy. This conclusion is supported by the results of the $1 \mathrm{D}$ test case too. It is possible to conclude from the results that one or two reactions are not enough to describe the ignition of hydrogen. Probably, the sophistication of abridged Jachimowski's model ( 7 reactions and $\mathrm{H}, \mathrm{O}$, and $\mathrm{OH}$ as intermediates) is a reasonable minimum for the modeling of hydrogen combustion in the high-temperature region $(T>$ $1000 \mathrm{~K}$ ). For the modeling in a wide-temperature range, the formation of $\mathrm{H}_{2} \mathrm{O}_{2}$ and $\mathrm{HO}_{2}$ should be taken into account. Of course, it would be very surprising to see that reduced or global mechanisms can describe ignition in a wide range of parameters. They are deduced from detailed mechanisms by neglecting the marginal processes, so they can not describe the behavior near margins. In most cases, the oxidation of hydrogen proceeds via the formation of $\mathrm{HO}_{2}$ radical, which is not included in the reduced mechanisms. In the new mechanism, the kinetics of $\mathrm{HO}_{2}$ is not comprehensive either.

It is necessary to note that shock tube is not a reactor with adiabatic solid walls. Due to the boundary layer effect, the temperature behind reflected shock wave slowly increases with time. The discrepancy between the ideal assumptions and reality arise at large-residence times, for the most of the shock tubes after $1 \mathrm{~ms}$. In Figure 2, where the ignition delay times are calculated using the boundary conditions of adiabatic solid walls, the detailed models have a "wrong" trend at low temperatures (large-residence times). At largeresidence time, it is necessary to take into account the real conditions behind reflected shock wave as it was done in [23]. In this case, the actual agreement between the experiments and the detailed models $[13,15,16]$ will be better than on the graph.

The performed simulations give more information about the evolution of the system than simply ignition delay times. The "classical" behavior was observed without anything unusual in all the " $0 \mathrm{D}$ " tests (by this reason, it is not included in the paper). All the kinetic models predict similar temperature (or pressure) time-resolved profiles, which have the induction period, the following temperature (or pressure) rise, which ends with asymptotic behavior. The 


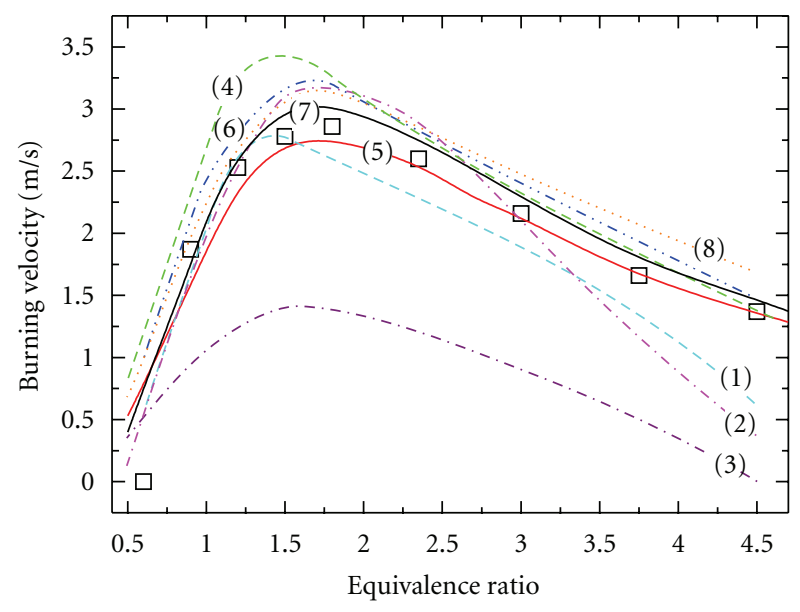

FIgURE 5: Burning velocities of a hydrogen-air mixture at $1 \mathrm{~atm}$. Squares experimental data [27]; (1) dash cyan line-Marinov et al. [11]; (2) dash dot magenta line-Lee and Kim [12]; (3) dash dot purple line-the abridged Jachimowski's model [8]; (4) dash line green-Zhukov (this work); (5) solid red line-O'Conaire et al. [13]; (6) dash dot dot line blue-Gutheil et al. [14]; (7) solid black line (the closest to exp. data)—Konnov [15]; (8) dot orange line-Burke et al. [16].

gas temperature (or pressure) of the combustion products is predicted correctly by all the kinetic models.

In the $1 \mathrm{D}$ test case the agreement of the simulating data with the experimental data is better overall than in the "ignition" case; see Figure 5. Practically all the models agree with the experimental data. The other distinctive feature of the obtained results is the bad agreement of abridged Jachimowski's model [8] and the good agreement of one-step model [11]. The results of 1D simulations can be interpretted in terms of (3). The system has practically the same physical properties in all the $1 \mathrm{D}$ simulations. These allow us to conclude that

$$
\frac{u_{1}}{u_{2}}=\sqrt{\frac{\tau_{2}}{\tau_{1}}}
$$

where indexes 1 and 2 designate the attribute to different kinetic models. The ignition delay times should be taken at flame temperature. In our case, the flame temperature amounts $\sim 2000 \mathrm{~K}$. Abridged Jachimowski's model [8] has the lowest effective activation energy among the models (see Figure 2) and predicts the significantly larger $\tau$ at high temperatures. As for the one-step model [11], which predicts the shortest $\tau$ at high temperatures, it does not include atomic hydrogen. It means that the assumptions, which lead us to (6), are not correct for this model. In terms of (3) neglecting of the diffusion hydrogen atom should lead to the smaller value of $\alpha$ and to the essentially less flame velocity, but it is compensated in this model by the small ignition delay time.

In Figures 2 and 5, we can see the difference between the results of the simulations and the experiments. While in the case of the global or reduced kinetic models, the discrepancy can be attributed to the weakness of models, the detailed

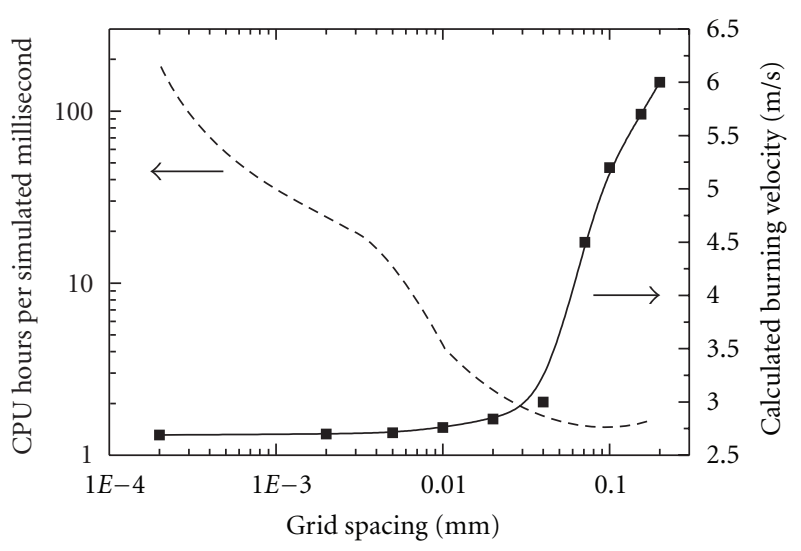

FIGURE 6: Simulating results and computational cost as the function of grid spacing. A hydrogen-air mixture at $1 \mathrm{~atm}$, kinetic model by Zhukov (this work).

reaction mechanisms [13-15] represent the state-of-the-art view on hydrogen kinetics. The agreement of these models with experimental data and the role of intermediates $(\mathrm{H}, \mathrm{O}$, $\mathrm{OH}, \mathrm{HO}_{2}$, and $\mathrm{H}_{2} \mathrm{O}_{2}$ ) were discussed in details in original articles [13-15]. The oxidation of hydrogen proceeds via the formation of highly active intermediates. The experimental study of the kinetics of the intermediates of $\mathrm{H}_{2} / \mathrm{O}_{2}$ system has some difficulties at temperatures below $900 \mathrm{~K}$ where in experiments, it is necessary to keep constant conditions during long-residence times. Thus, even a detailed kinetic scheme can fail near ignition and flammability limits.

Numerical parameters such as time step, grid spacing, and type of difference scheme should not determine the results of modeling. The proper values of time step and grid spacing should correspond via the coefficients of physical model to physical time and space scales. On practical ground, the upper limits of time step and grid spacing are more important, because computational cost is generally inversely proportional to time step and the amount of nodes (for the employed grid the amount of nodes is reciprocally proportional to the grid spacing); see Figure 6. The employed values of time step and grid spacing are normally close to the upper limit. Generally chemical processes can not be faster than several fractions of microsecond, and hydrodynamic processes can not take place on a scale smaller than a micrometer. Thus, these values can be set as a reasonable lower limit for the time step and the grid spacing. The upper limit is quite specific to the details of a task. It is necessary to estimate the maximum time and mesh steps in each case separately. At a too high time step, the solution diverges. Numerical noise and residuals can be used as the measure of the proximity to the upper limit of time step. In this work, the adaptive time step has been used and it has been defined by a residual. The time step was decreased or increased until the value of the residuals reached the desired level. To estimate the upper limit of mesh step, several simulations were carried out with different spacing; see Figure 6. On the plot, we can see a plateau for the cell size below $5 \mu \mathrm{m}$. The upper limit, which is located near $10 \mu \mathrm{m}$, is related to the flame thickness. 
TABle 3: Parameters of kinetic models and the computational costs. Case 1: test case "ignition," 38 cells; case 2: test case "flame propagation," 400 cells.

\begin{tabular}{|c|c|c|c|c|}
\hline Model & Number of equations* & Number of species & $\begin{array}{c}\text { Case } 1, \mathrm{CPU} \\
\text { hours/ms }\end{array}$ & $\begin{array}{c}\text { Case } 2, \mathrm{CPU} \\
\text { hours/ms }\end{array}$ \\
\hline Marinov et al. [11] & 1 & $3\left(\mathrm{H}_{2}, \mathrm{O}_{2}, \mathrm{H}_{2} \mathrm{O}\right)+\mathrm{N}_{2}$ & 0.18 & 0.14 \\
\hline Lee and Kim [12] & 2 & $4(3+H)+$ bath gas & 0.43 & 0.20 \\
\hline $\begin{array}{l}\text { abridged Jachimowski's } \\
\text { model [8] }\end{array}$ & 7 & $6(3+\mathrm{H}, \mathrm{O}, \mathrm{OH})+$ bath gas & 1.7 & 5.2 \\
\hline Zhukov (this work) & 13 & $8+$ bath gas & 1.6 & 19 \\
\hline Gutheil et al. [14] & 21 & $8+$ bath gas & 2.5 & 67 \\
\hline O'Conaire et al. [13] & 23 & $8+$ bath gas & 1.8 & 71 \\
\hline Konnov [15] & 29 & $8+$ bath gases & 2.3 & 36 \\
\hline Burke et al. [16] & 22 & $8+$ bath gases & 1.8 & 35 \\
\hline
\end{tabular}

${ }^{*}$ The number of equations could exceed the number of reactions because of the possible presence of double reactions and of third body reactions where the activation energy depends on the collisional partner.

In flame front, the concentration of hydrogen increases in 2 4 times each $10 \mu \mathrm{m}$. Probably the maximum grid spacing is universal for all hydrogen-air flames at $1 \mathrm{~atm}$ and is defined by the transport properties of the system while the maximum time step is individual for each kinetic model. As we can see later, maximum time step is related to the stiffness of kinetic scheme.

Grid spacing, the physical dimensions of computational domain, and computational cost are connected with each other. At these conditions, grid spacing can limit the applicability of kinetic model. In Table 3 the computational costs, which are required for the simulation of the evolution of the system during $1 \mathrm{~ms}$ on $1 \mathrm{CPU}$ (Pentium 4) at $2 \mathrm{GHz}$, are presented for all the tested models. In the identical conditions, computational cost varies by orders from one model to another. It is impossible from the data of Table 3 to see any direct connection of the computational cost with the number of reactions and species. However, there is a trend: detailed kinetic models require a much more computational power than reduced models. Thus, the high computational cost limits the application of detailed kinetic mechanisms in CFD calculations seriously. The grid spacing has the limit near $40 \mu \mathrm{m}$ after which the simulations give the absolutely unrealistic results. Detailed models can be employed only in a special tasks with the computational domains of small sizes due to the high computational cost and the small grid spacing.

The computational cost increases strongly from the global reaction models to the detailed kinetic mechanisms, but the number of chemical equations and species does not completely determine computation cost. Another essential parameter is the stiffness of kinetic model. Stiffness is the embedded parameter of each kinetic model. It determines the maximum time step during calculations. It arises due to differences in timescales for different chemical reactions. To solve kinetic equations it necessary to integrate equations using time step related the smallest timescale over the time interval related to the largest timescale. Stiffness can be characterized the ratio of timescales. For example, in preflame zone reaction (R9) is in $10^{10}$ times faster than reaction (R1) while in postflame zone, it is vice versa, reaction (R1) is faster than reaction (R9) in 100 times. It is typical for combustion problems, when important fast reactions run on the background of slow equally important processes. Another example of the embedded stiffness is reaction:

$$
\mathrm{H}_{2} \mathrm{O}_{2}+\mathrm{M} \longrightarrow \mathrm{OH}+\mathrm{OH}+\mathrm{M} .
$$

This reaction determines concentration of $\mathrm{H}_{2} \mathrm{O}_{2}$ in flame, and it changes its direction from reverse to forward after passing the flame front.

\section{Conclusions}

The eight different kinetic models of hydrogen oxidation were verified, validated, and tested in the CFD simulations what was done using ANSYS CFX 11 software. The two cases: ignition in adiabatic constant volume reactor and the propagation of free laminar flame were considered. The verification of the kinetic models was done through the comparison with the results obtained with the help of the Chemkin software. The verification allowed to eliminate misentries and to define correctly the thermodynamic, kinetic, and transport properties.

The subsequent validation showed that the detailed kinetic schemes are more accurate than the reduced ones. While it was not found any dependence between the "speed" of kinetic model and the number of reaction and species, the reduced kinetic schemes are faster than the detailed ones. The simulations showed the common trend for kinetic models: more details—-higher computational cost-higher precision. The simulation of the ignition of hydrogen-air mixture showed that the results are sensitive to the choice of kinetic model. However, in the case of the flame propagation the results are more sensitive to the model of the transport properties while the reasonable results can be achieved even with the use of global reaction mechanism.

The comparison of the simulating data with the experimental data $[10,27]$ showed that the detailed kinetic schemes $[13,15,16]$ agree with experiments well, while the 
nondetailed schemes agree with the experiments only within a limited range. The kinetic model by Konnov [15] has the best agreement with the experimental data among the tested models. The application of reduced kinetic schemes of hydrogen combustion, which do not take into account chemical reactions with $\mathrm{HO}_{2}$ and $\mathrm{H}_{2} \mathrm{O}_{2}$, is possible only with strong limitations.

For the debugging purposes, the new skeletal kinetic scheme was developed which represents the good compromise between computational cost and accuracy.

The carried out study showed that computational results are affected by the parameters of physical and numerical models. A large amount of model parameters is the potential source of errors. The number of different coefficients reaches thousands in the simulation with the use of a detailed kinetic model. The parameters of the model can be verified and validated using the proposed method.

The application of kinetic models in CFD calculations requires the considerable amount of computational power. The maximum time step is limited by the stiffness of model and alters from model to model while the maximum grid spacing is more or less universal and defined by the thickness of flame front.

\section{Acknowledgments}

The author is grateful to Andreas Gernoth for introducing me into ANSYS CFX. Also, the author appreciates the scientific discussions with Dr. Oskar Haidn.

\section{References}

[1] ANSYS CFX, http://www.ansys.com/products/cfx.asp.

[2] Fluent, http://www.fluent.com/.

[3] Star-CD, http://www.cd-adapco.com/products/STAR-CD/.

[4] P. J. Roache, "Verification of codes and calculations," AIAA Journal, vol. 36, no. 5, pp. 696-702, 1998.

[5] R. J. Kee, F. M. Rupley, and J. A. Miller, "CHEMKIN-II: a fortran chemical kinetics package for the analysis of gasphase chemical kinetics," Sandia National Laboratories Report SAND898009, 1989.

[6] M. Mani, R. H. Bush, and P. G. Vogel, "Implicit equilibrium and finite-rate chemistry models for high speed flow applications," AIAA Paper 91-3299-CP, 1991.

[7] C. J. Jachimowski, "An analytical study of hydrogen-air reaction mechanism with application to scramjet," NASA Technical Paper 2791, 1988.

[8] D. R. Eklund and S. D. Stouffer, "A numerical and experimental study of a supersonic combustor employing swept ramp fuel injectors," AIAA Paper 94-2819, 1994.

[9] K. Kumaran and V. Babu, "Investigation of the effect of chemistry models on the numerical predictions of the supersonic combustion of hydrogen," Combustion and Flame, vol. 156, no. 4, pp. 826-841, 2009.

[10] M. Slack and A. Grillo, "Investigation of hydrogen-air ignition sensitized by nitric oxide and by nitrogen oxide," NASA Report CR-2896, 1977.

[11] N. M. Marinov, C. K. Westbrook, and W. J. Pitz, Detailed and Global Chemical Kinetic Model for Hydrogen, vol. 1, Transport Phenomena in Combustion, Washington, DC, USA, 1996.
[12] S. R. Lee and J. S. Kim, "Structure and length of chemistry induction zone in hydrogen-air detonations," Korean Journal of Chemical Engineering, vol. 16, no. 2, pp. 253-259, 1999.

[13] M. O'Conaire, H. J. Curran, J. M. Simmie, W. J. Pitz, and C. K. Westbrook, "A comprehensive modeling study of hydrogen oxidation," International Journal of Chemical Kinetics, vol. 36, no. 11, pp. 603-622, 2004.

[14] E. Gutheil, M. D. Balakrishnan, and F. A. Williams, "Structure and extinction of hydrogen-air diffusion flames," in Reduced Mechanisms for Application in Combustion Systems, vol. 15, pp. 177-195, Springer, Heidelberg, Germany, 1993.

[15] A. A. Konnov, "Development and validation of a detailed reaction mechanism for the combustion of small hydrocarbons," in Proceedings of the 28th International Symposium on Combustion, Abstract Symposium Paper, p. 317, August 2000.

[16] M. P. Burke, M. Chaos, Y. Ju, F. L. Dryer, and S. J. Klippenstein, "Comprehensive $\mathrm{H}_{2} / \mathrm{O}_{2}$ kinetic model for high-pressure combustion," International Journal of Chemical Kinetics, vol. 44, no. 7, pp. 444-474, 2012.

[17] G. Stahl and J. Warnatz, "Numerical investigation of timedependent properties and extinction of strained methane- and propane-air flamelets," Combustion and Flame, vol. 85, no. 34, pp. 285-299, 1991.

[18] J. V. Michael, M. C. Su, J. W. Sutherland, J. J. Carroll, and A. F. Wagner, "Rate constants for $\mathrm{H}+\mathrm{O}_{2}+\mathrm{M} \rightarrow \mathrm{HO}_{2}+\mathrm{M}$ in seven bath gases," The Journal of Physical Chemistry A, vol. 106, no. 21, pp. 5297-5313, 2002.

[19] B. Ruscic, A. F. Wagner, L. B. Harding et al., "On the enthalpy of formation of hydroxyl radical and gas-phase bond dissociation energies of water and hydroxyl," Journal of Physical Chemistry A, vol. 106, no. 11, pp. 2727-2747, 2002.

[20] A. A. Konnov, "Remaining uncertainties in the kinetic mechanism of hydrogen combustion," Combustion and Flame, vol. 152, no. 4, pp. 507-528, 2008.

[21] J. Li, Z. Zhao, A. Kazakov, and F. L. Dryer, "An updated comprehensive kinetic model of hydrogen combustion," International Journal of Chemical Kinetics, vol. 36, no. 10, pp. 566$575,2004$.

[22] O. P. Shatalov, L. B. Ibraguimova, V. A. Pavlov et al., "Analysis of the kinetic data described oxygen-hydrogen mixtures combustion," in Proceedings of the 4th European Combustion Meeting, Vienna, Austria, April 2009.

[23] Z. Hong, D. F. Davidson, and R. K. Hanson, "An improved $\mathrm{H}_{2} / \mathrm{O}_{2}$ mechanism based on recent shock tube/laser absorption measurements," Combustion and Flame, vol. 158, no. 4, pp. 633-644, 2011.

[24] M. P. Burke, M. Chaos, F. L. Dryer, and Y. Ju, "Negative pressure dependence of mass burning rates of $\mathrm{H}_{2} / \mathrm{CO} / \mathrm{O}_{2} /$ diluent flames at low flame temperatures," Combustion and Flame, vol. 157, no. 4, pp. 618-631, 2010.

[25] P. Gerlinger, K. Nold, and M. Aigner, "Influence of reaction mechanisms, grid spacing, and inflow conditions on the numerical simulation of lifted supersonic flames," International Journal for Numerical Methods in Fluids, vol. 62, no. 12, pp. 1357-1380, 2010.

[26] P. K. Tucker, S. Menon, C. L. Merkle, J. C. Oefelein, and V. Yang, "Validation of high-fidelity CFD simulations for rocket injector design," in Proceedings of the 44th AIAA/ASME/SAE/ASEE Joint Propulsion Conference and Exhibit, vol. 5226, AIAA, Hartford, Conn, USA, July 2008.

[27] O. C. Kwon and G. M. Faeth, "Flame/stretch interactions of premixed hydrogen-fueled flames: measurements and predictions," Combustion and Flame, vol. 124, no. 4, pp. 590-610, 2001. 
[28] O. Gurliat, V. Schmidt, O. J. Haidn, and M. Oschwald, "Ignition of cryogenic $\mathrm{H}_{2}$ /LOX sprays," Aerospace Science and Technology, vol. 7, no. 7, pp. 517-531, 2003.

[29] D. Suslov, J. Lux, and O. Haidn, "Investigation of porous injector elements for $\mathrm{LOX} / \mathrm{CH}_{4}$ and $\mathrm{LOX} / \mathrm{H}_{2}$ combustion at sub-and super-critical conditions," in Proceedings of the 2nd European Conference for Aerospace Sciences, pp. 1-6, Brussels, Belgium, July 2007.

[30] T. G. Kreutz and C. K. Law, "Ignition in nonpremixed counterflowing hydrogen versus heated air: computational study with skeletal and reduced chemistry," Combustion and Flame, vol. 114, no. 3-4, pp. 436-456, 1998.

[31] V. P. Zhukov, "Kinetic model of alkane oxidation at high pressure from methane to $n$-heptane," Combustion Theory and Modelling, vol. 13, no. 3, pp. 427-442, 2009.

[32] R. J. Kee, J. F. Grcar, M. D. Smooke, and J. A. Miller, "A fortran program for modeling steady laminar onedimensional premixed flames," Sandia National Laboratories Report SAND89-8240, 1985.

[33] J. Warnatz, U. Mass, and R. W. Dibble, Combustion, Springer, New York, NY, USA, 1996.

[34] G. Dixon-Lewis, "Flame structure and flame reaction kinetics. II. transport phenomena in multicomponent systems," Proceedings of the Royal Society, vol. 307, no. 1488, pp. 111-135, 1968.

[35] T. P. Coffee and J. M. Heimerl, "Transport algorithms for premixed, laminar steady-state flames," Combustion and Flame, vol. 43, pp. 273-289, 1981.

[36] F. Yang, C. K. Law, C. J. Sung, and H. Q. Zhang, "A mechanistic study of soret diffusion in hydrogen-air flames," Combustion and Flame, vol. 157, no. 1, pp. 192-200, 2010. 

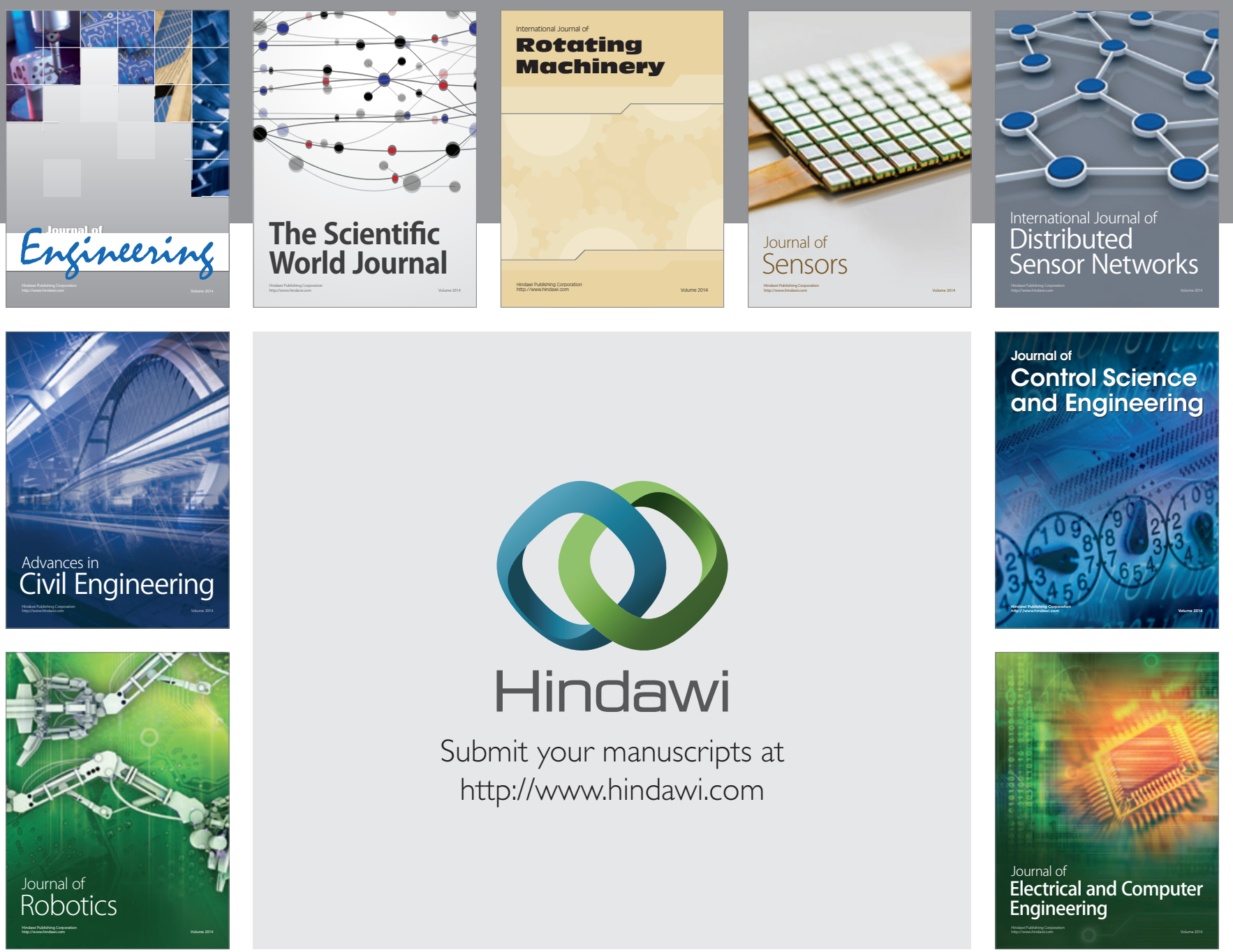

Submit your manuscripts at

http://www.hindawi.com
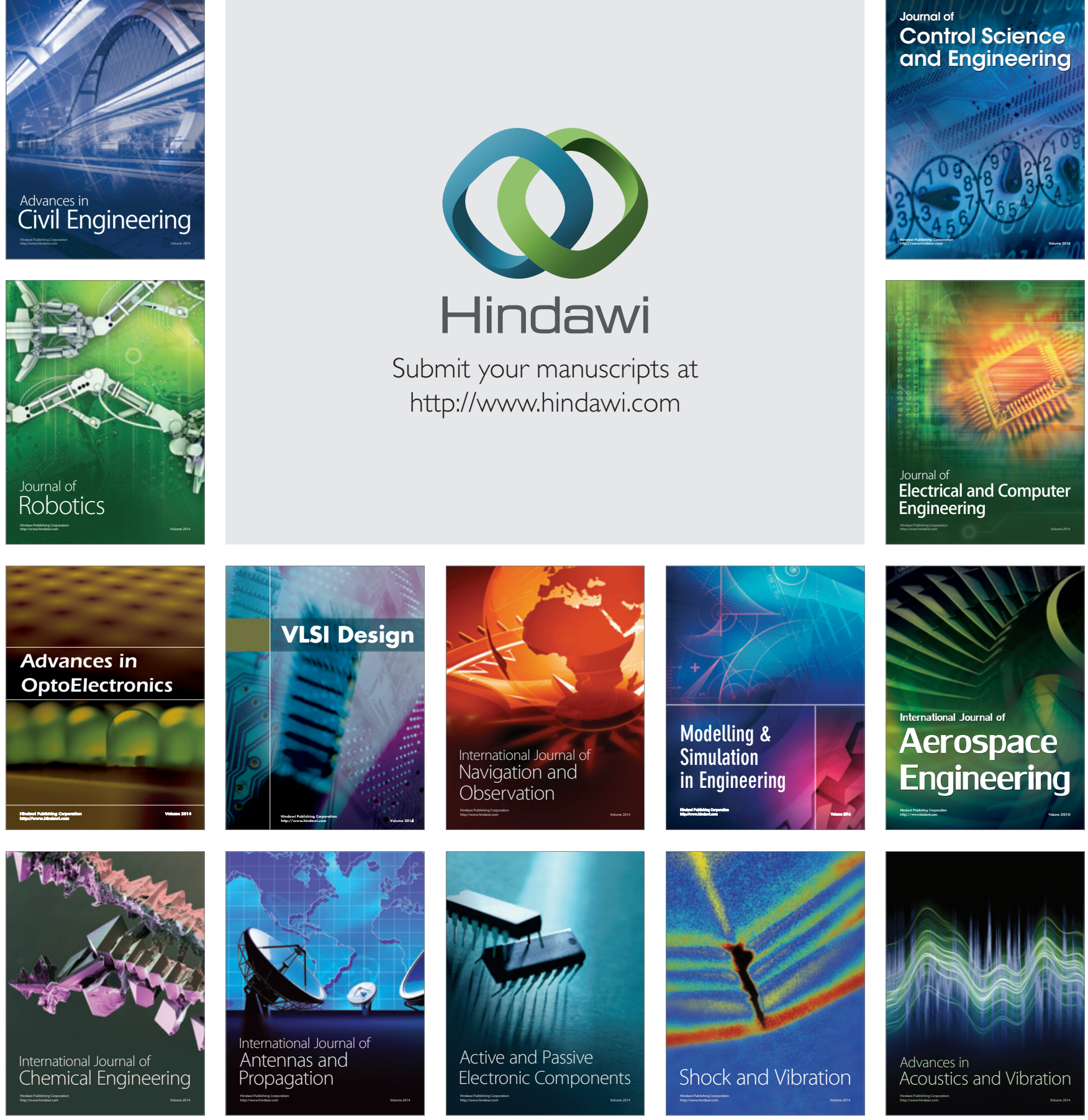